\title{
Symmetry Analysis and Conservation Laws of a Generalized Two-Dimensional Nonlinear KP-MEW Equation
}

\author{
Khadijo Rashid Adem and Chaudry Masood Khalique \\ International Institute for Symmetry Analysis and Mathematical Modelling, Department of Mathematical Sciences, \\ North-West University, Mafikeng Campus, Private Bag X 2046, Mmabatho 2735, South Africa \\ Correspondence should be addressed to Chaudry Masood Khalique; masood.khalique@nwu.ac.za
}

Received 2 August 2014; Accepted 20 September 2014

Academic Editor: Hossein Jafari

Copyright (C) 2015 K. R. Adem and C. M. Khalique. This is an open access article distributed under the Creative Commons Attribution License, which permits unrestricted use, distribution, and reproduction in any medium, provided the original work is properly cited.

\begin{abstract}
Lie symmetry analysis is performed on a generalized two-dimensional nonlinear Kadomtsev-Petviashvili-modified equal width equation. The symmetries and adjoint representations for this equation are given and an optimal system of one-dimensional subalgebras is derived. The similarity reductions and exact solutions with the aid of $\left(G^{\prime} / G\right)$-expansion method are obtained based on the optimal systems of one-dimensional subalgebras. Finally conservation laws are constructed by using the multiplier method.
\end{abstract}

\section{Introduction}

Nonlinear evolution equations (NLEEs) have been widely used to describe natural phenomena of science and engineering. Therefore it is very important to find exact solutions of NLEEs. However, this is not an easy task. During the past few decades various integration techniques have been developed by the researchers to solve these NLEEs. Some of the well-known techniques used in the literature are the inverse scattering transform method [1], the homogeneous balance method [2], the Bäcklund transformation [3], the Weierstrass elliptic function expansion method [4], the Darboux transformation [5], the ansatz method [6, 7], Hirota's bilinear method [8], the $\left(G^{\prime} / G\right)$-expansion method [9], the Jacobi elliptic function expansion method [10,11], the variable separation approach [12], the sine-cosine method [13], the trifunction method [14,15], the F-expansion method [16], the exp-function method [17], the multiple exp-function method [18], and the Lie symmetry method [19-25].

The purpose of this paper is to study one such NLEE, namely, the generalized two-dimensional nonlinear Kadomtsev-Petviashvili-modified equal width (KP-MEW) equation [26] that is given by

$$
\left(u_{t}+\alpha\left(u^{n}\right)_{x}+\beta u_{x x t}\right)_{x}+\gamma u_{y y}=0
$$

Here, in (1) $\alpha, \beta, \gamma$ and $n>1$ are real valued constants. The solutions of (1) have been studied in various aspects. See, for example, the recent papers [26-28]. Wazwaz [26] used the tanh method and the sine-cosine method, for finding solitary waves and periodic solutions. Saha [27] used the theory of bifurcations of planar dynamical systems to prove the existence of smooth and nonsmooth travelling wave solutions. Wei et al. [28] used the qualitative theory of differential equations and obtained peakon, compacton, cuspons, loop soliton solutions, and smooth soliton solutions.

In this paper we obtain symmetry reductions of (1) using Lie group analysis [19-24] and based on the optimal systems of one-dimensional subalgebras. Furthermore, the $\left(G^{\prime} / G\right)$ expansion method is employed to obtain some exact solutions of (1). In addition to this conservation laws will be derived for (1) using the multiplier method [29].

\section{Symmetry Reductions and Exact Solutions of (1)}

The vector field of the form

$$
X=\xi^{1} \frac{\partial}{\partial x}+\xi^{2} \frac{\partial}{\partial y}+\xi^{3} \frac{\partial}{\partial t}+\eta \frac{\partial}{\partial u}
$$


where $\xi^{i}, i=1,2,3$, and $\eta$ depend on $x, y, t$, and $u$, is a Lie point symmetry of (1) if

$$
\operatorname{pr}^{(4)} X\left[\left(u_{t}+\alpha\left(u^{n}\right)_{x}+\beta u_{x x t}\right)_{x}+\gamma u_{y y}\right]=0
$$

whenever $\left(u_{t}+\alpha\left(u^{n}\right)_{x}+\beta u_{x x t}\right)_{x}+\gamma u_{y y}=0$. Here $\operatorname{pr}^{(4)} X$ [20] denotes the fourth prolongation of $X$. Expanding (3) and splitting on the derivatives of $u$, we obtain an overdetermined system of linear partial differential equations. Solving this system one obtains the following four Lie point symmetries:

$$
\begin{aligned}
& X_{1}=\frac{\partial}{\partial x}, \\
& X_{2}=\frac{\partial}{\partial y}, \\
& X_{3}=\frac{\partial}{\partial t}, \\
& X_{4}=y(1-n) \frac{\partial}{\partial y}+2 t(1-n) \frac{\partial}{\partial t}+2 u \frac{\partial}{\partial u} .
\end{aligned}
$$

2.1. One-Dimensional Optimal System of Subalgebras. We now calculate the optimal system of one-dimensional subalgebras for (1) and use it to find the optimal system of groupinvariant solutions for (1). We follow the method given in [20]. Recall that the adjoint transformations are given by

$$
\begin{aligned}
\operatorname{Ad}\left(\exp \left(\epsilon X_{i}\right)\right) X_{j}= & X_{j}-\epsilon\left[X_{i}, X_{j}\right] \\
& +\frac{1}{2} \epsilon^{2}\left[X_{i}\left[X_{i}, X_{j}\right]\right]-\cdots,
\end{aligned}
$$

where $\left[X_{i}, X_{j}\right]$ is the commutator defined by

$$
\left[X_{i}, X_{j}\right]=X_{i} X_{j}-X_{j} X_{i}
$$

We present the commutator table of the Lie symmetries and the adjoint representations of the symmetry group of (1) on its Lie algebra in Tables 1 and 2, respectively. These two tables are then used to construct the optimal system of one-dimensional subalgebras for (1). As a result, after some calculations, one can obtain an optimal system of onedimensional subalgebras given by $\left\{a X_{1}+b X_{2}+c X_{3}, d X_{1}+X_{4}\right\}$, where $a, d \in \mathbb{R}, b, c=0, \pm 1$.

2.2. Symmetry Reductions and Exact Solutions of (1). In this subsection we use the optimal system of one-dimensional subalgebras calculated above to obtain symmetry reductions and exact solutions of the KP-MEW equation.

Case 1. Consider the following: $a X_{1}+b X_{2}+c X_{3} ; a \in \mathbb{R}, b, c=$ \pm 1 .

The symmetry $a X_{1}+b X_{2}+c X_{3}$ gives rise to the following three invariants:

$$
f=\frac{b t-c y}{b}, \quad g=\frac{b x+a y}{b}, \quad \phi=u .
$$

TABLE 1: Commutator table of the Lie algebra of equation (1).

\begin{tabular}{ccccc}
\hline & $X_{1}$ & $X_{2}$ & $X_{3}$ & $X_{4}$ \\
\hline$X_{1}$ & 0 & 0 & 0 & 0 \\
$X_{2}$ & 0 & 0 & 0 & $(1-n) X_{2}$ \\
$X_{3}$ & 0 & 0 & 0 & $(2-2 n) X_{3}$ \\
$X_{4}$ & 0 & $-(1-n) X_{2}$ & $-(2-2 n) X_{3}$ & 0 \\
\hline
\end{tabular}

TABLE 2: Adjoint table of the Lie algebra of equation (1).

\begin{tabular}{ccccc}
\hline Ad & $X_{1}$ & $X_{2}$ & $X_{3}$ & $X_{4}$ \\
\hline$X_{1}$ & $X_{1}$ & $X_{2}$ & $X_{3}$ & $X_{4}$ \\
$X_{2}$ & $X_{1}$ & $X_{2}$ & $X_{3}$ & $X_{4}-\varepsilon(1-n) X_{2}$ \\
$X_{3}$ & $X_{1}$ & $X_{2}$ & $X_{3}$ & $X_{4}-\varepsilon(2-2 n) X_{3}$ \\
$X_{4}$ & $X_{1}$ & $e^{\varepsilon(1-n)} X_{2}$ & $e^{\varepsilon(2-2 n)} X_{3}$ & $X_{4}$ \\
\hline
\end{tabular}

Now treating $\phi$ as the new dependent variable and $f$ and $g$ as new independent variables, the KP-MEW equation (1) transforms to

$$
\begin{gathered}
\left(2 a c \gamma+b^{2}\right) \phi_{f g}+a^{2} \gamma \phi_{g g}+\alpha n\left(n-b^{2}\right) \phi^{n-2} \phi_{g}^{2} \\
+b^{2} \alpha n \phi^{n-1} \phi_{g g}+b^{2} \beta \phi_{f g g g}+c^{2} \gamma \phi_{f f}=0
\end{gathered}
$$

which is a nonlinear PDE in two independent variables. We now use the Lie point symmetries of (8) and transform it to an ordinary differential equation (ODE). Equation (8) has the two translational symmetries; namely,

$$
\Gamma_{1}=\frac{\partial}{\partial f}, \quad \Gamma_{2}=\frac{\partial}{\partial g} .
$$

The combination $\Gamma_{1}+\Gamma_{2}$ of the two symmetries $\Gamma_{1}$ and $\Gamma_{2}$ yields the two invariants

$$
z=f-g, \quad F=\phi,
$$

which gives rise to a group-invariant solution $F=F(z)$. Consequently using these invariants, (8) is transformed into the fourth-order nonlinear ODE:

$$
\begin{aligned}
& \gamma(a+c)^{2} F^{\prime \prime}+\alpha n b^{2} F^{n-1} F^{\prime \prime}-b^{2} F^{\prime \prime} \\
& \quad+\alpha n b^{2}(n-1) F^{n-2} F^{\prime 2}-\beta b^{2} F^{\prime \prime \prime \prime}=0 .
\end{aligned}
$$

Integrating the above equation twice and taking the constants of integration to be zero we obtain a second-order ODE:

$$
\left(\gamma(a+c)^{2}-c^{2}\right) F+\alpha b^{2} F^{n}-\beta b^{2} F^{\prime \prime}=0 .
$$

Multiplying (12) by $F^{\prime}$, integrating once and taking the constant of integration to be zero, we obtain the first-order ODE:

$$
\frac{1}{2}\left(\gamma(a+c)^{2}-b^{2}\right) F^{2}+\frac{\alpha b^{2}}{n+1} F^{n+1}-\frac{1}{2} \beta b^{2} F^{\prime 2}=0 .
$$

One can integrate the above equation by separating the variables. After integrating and reverting back to the original variables, we obtain the following group-invariant solutions 
of the KP-MEW equation (1) for arbitrary values of $n$ in the following form:

$$
\begin{aligned}
& u(x, y, t) \\
& \quad=\left(\frac{(n+1)\left(b^{2}-\gamma(a+c)^{2}\right)}{2 \beta b^{2}}\right)^{1 /(n-1)} \operatorname{sech}^{2 /(n-1)}[Q],
\end{aligned}
$$

where

$$
\begin{aligned}
& Q=\frac{\sqrt{\gamma(a+c)^{2}+b^{2}}}{2 \sqrt{2}}\left(\frac{\sqrt{2}(1-n)}{c \sqrt{\beta}} z+(n-1) C\right), \\
& z=t-x-\frac{(a+c)}{b} y,
\end{aligned}
$$

and $C$ is a constant of integration. By taking $n=2, \alpha=1 / 2$, $\beta=1, \gamma=1, a=1, b=1, c=1, t=0$, and $C=1$ in (14), the profile of the solution is given in Figure 1.

Case 2. Consider the following: $d X_{1}+X_{4}$.

The symmetry $d X_{1}+X_{4}$ gives rise to the three invariants:

$$
f=\frac{t}{y^{2}}, \quad g=\frac{d \ln y+n x-x}{n-1}, \quad \phi=u y^{2 /(n-1)} \text {. }
$$

By treating $\phi$ as the new dependent variable and $f$ and $g$ as new independent variables, the KP-MEW equation (1) transforms to

$$
\begin{gathered}
\beta n^{2} \phi_{f g g g}-2 \beta n \phi_{f g g g}+2 d \gamma \phi_{g g}+2 n \gamma \phi+4 f^{2} \gamma \phi_{f f} \\
-2 f \gamma \phi_{f}-3 a_{1} \gamma \phi_{g}+\phi_{f g}-\alpha n \phi^{n-2} \phi_{g}^{2} \\
+\alpha n^{4} \phi^{n-2} \phi_{g}^{2}+3 \alpha n^{2} \phi^{n-2} \phi_{g}^{2}+6 f n^{2} \gamma \phi_{f} \\
\quad-4 f n \gamma \phi_{f}-3 \alpha n^{3} \phi^{n-2} \phi_{g}^{2}+\alpha n \phi^{n-1} \phi_{g g} \\
+\alpha n^{3} \phi^{n-1} \phi_{g g}-8 f^{2} n \gamma \phi_{f f}+4 f^{2} n^{2} \gamma \phi_{f f} \\
+4 d f \gamma \phi_{f g}-2 \alpha n^{2} \phi^{n-1} \phi_{g g}+2 \gamma \phi+\beta \phi_{f g g g} \\
+f n^{2} \phi_{g}-2 f n \phi_{g}-4 d f n \gamma \phi_{f g}-d f n \gamma \phi_{g}=0 .
\end{gathered}
$$

Equation (17) has a single Lie point symmetry; namely,

$$
\Gamma_{1}=\frac{\partial}{\partial g}
$$

and this symmetry yields the two invariants

$$
r=f, \quad F=\phi,
$$

which gives rise to a group-invariant solution $F=F(r)$ and consequently, using these invariants, (17) is then transformed to a second-order Cauchy-Euler ODE:

$$
\begin{aligned}
& \left(2 n^{2} r^{2}-4 n r^{2}+2 r^{2}\right) F^{\prime \prime}+\left(3 n^{2} r-2 n r-r\right) F^{\prime} \\
& +(1+n) F=0 .
\end{aligned}
$$

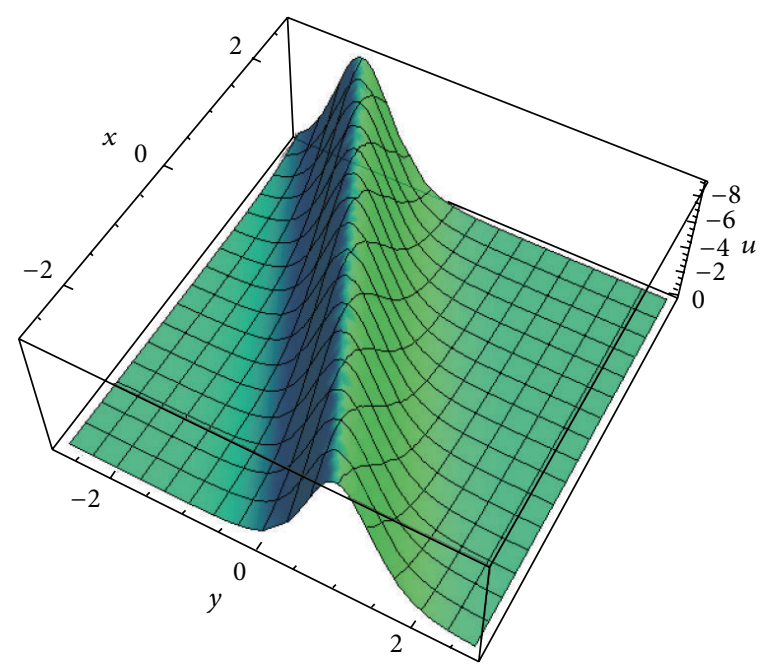

Figure 1: Profile of solution (14).

Now solving this equation and reverting back to the original variables, we obtain the following solution of the KP-MEW equation (1):

$$
u(x, y, t)=\frac{1}{y^{2 /(n-1)}}\left(C_{1} r^{-(n+1) / 2(n-1)}+C_{2} r^{-1 /(n-1)}\right),
$$

where $r=t / y^{2}$ and $C_{1}$ and $C_{2}$ are constants of integration.

\section{3. $\left(G^{\prime} / G\right)$-Expansion Method}

In this section we use the $\left(G^{\prime} / G\right)$-expansion method $[9,30]$ to obtain a few exact solutions of the KP-MEW equation (1) for $n=2$ and $n=3$.

Let us consider the solutions of (11) in the form

$$
F(z)=\sum_{i=0}^{M} \mathscr{A}_{i}\left(\frac{G^{\prime}(z)}{G(z)}\right)^{i}
$$

where $G(z)$ satisfies

$$
G^{\prime \prime}+\lambda G^{\prime}+\mu G=0
$$

and $\lambda$ and $\mu$ are constants. The homogeneous balance method between the highest order derivative and highest order nonlinear term appearing in (11) determines the value of $M$ and $\mathscr{A}_{0}, \ldots, \mathscr{A}_{M}$ are constants to be determined.

Consider $n=2$. Application of the balancing procedure to fourth-order ODE (11) yields $M=2$, so the solution of (11) is of the form

$$
F(z)=\mathscr{A}_{0}+\mathscr{A}_{1}\left(\frac{G^{\prime}(z)}{G(z)}\right)+\mathscr{A}_{2}\left(\frac{G^{\prime}(z)}{G(z)}\right)^{2} .
$$


Substituting (23) and (24) into (11) leads to an overdetermined system of algebraic equations. Solving this system of algebraic equations with the aid of Maple, we obtain

$$
\begin{aligned}
& \mathscr{A}_{0}=\frac{b^{2} \alpha \lambda^{2} A_{2}+8 b^{2} \mu \alpha A_{2}-6 \gamma a^{2}-12 a c \gamma-6 \gamma c^{2}+6 b^{2}}{12 \alpha b^{2}}, \\
& \mathscr{A}_{1}=\frac{6 \beta \lambda}{\alpha}, \\
& \mathscr{A}_{2}=\frac{6 \beta}{\alpha} .
\end{aligned}
$$

Now using the general solution of (23) in (24), we have the following three types of travelling wave solutions of the KPMEW equation (1).

When $\lambda^{2}-4 \mu>0$, we obtain the hyperbolic function solution:

$$
\begin{aligned}
u(x, y, t) & \\
= & \mathscr{A}_{0}+\mathscr{A}_{1}\left(-\frac{\lambda}{2}+\delta_{1} \frac{C_{1} \sinh \left(\delta_{1} z\right)+C_{2} \cosh \left(\delta_{1} z\right)}{C_{1} \cosh \left(\delta_{1} z\right)+C_{2} \sinh \left(\delta_{1} z\right)}\right) \\
& +\mathscr{A}_{2}\left(-\frac{\lambda}{2}+\delta_{1} \frac{C_{1} \sinh \left(\delta_{1} z\right)+C_{2} \cosh \left(\delta_{1} z\right)}{C_{1} \cosh \left(\delta_{1} z\right)+C_{2} \sinh \left(\delta_{1} z\right)}\right)^{2},
\end{aligned}
$$

where $z=t-x-((a+c) / b) y, \delta_{1}=(1 / 2) \sqrt{\lambda^{2}-4 \mu}$, and $C_{1}$ and $C_{2}$ are arbitrary constants.

The profile of the solution (26) is given in Figure 2.

When $\lambda^{2}-4 \mu<0$, we obtain the trigonometric function solution:

$$
\begin{aligned}
u(x, y, t) & \\
= & \mathscr{A}_{0}+\mathscr{A}_{1}\left(-\frac{\lambda}{2}+\delta_{2} \frac{-C_{1} \sin \left(\delta_{2} z\right)+C_{2} \cos \left(\delta_{2} z\right)}{C_{1} \cos \left(\delta_{2} z\right)+C_{2} \sin \left(\delta_{2} z\right)}\right) \\
& +\mathscr{A}_{2}\left(-\frac{\lambda}{2}+\delta_{2} \frac{-C_{1} \sin \left(\delta_{2} z\right)+C_{2} \cos \left(\delta_{2} z\right)}{C_{1} \cos \left(\delta_{2} z\right)+C_{2} \sin \left(\delta_{2} z\right)}\right)^{2},
\end{aligned}
$$

where $z=t-x-((a+c) / b) y, \delta_{2}=(1 / 2) \sqrt{4 \mu-\lambda^{2}}$, and $C_{1}$ and $C_{2}$ are arbitrary constants.

The profile of the solution (27) is given in Figure 3.

When $\lambda^{2}-4 \mu=0$, we obtain the rational function solution:

$$
\begin{aligned}
u(x, y, t)= & \mathscr{A}_{0}+\mathscr{A}_{1}\left(-\frac{\lambda}{2}+\frac{C_{2}}{C_{1}+C_{2} z}\right) \\
& +\mathscr{A}_{2}\left(-\frac{\lambda}{2}+\frac{C_{2}}{C_{1}+C_{2} z}\right)^{2},
\end{aligned}
$$

where $z=t-x-((a+c) / b) y$ and $C_{1}$ and $C_{2}$ are arbitrary constants.

The profile of the solution (28) is given in Figure 4.

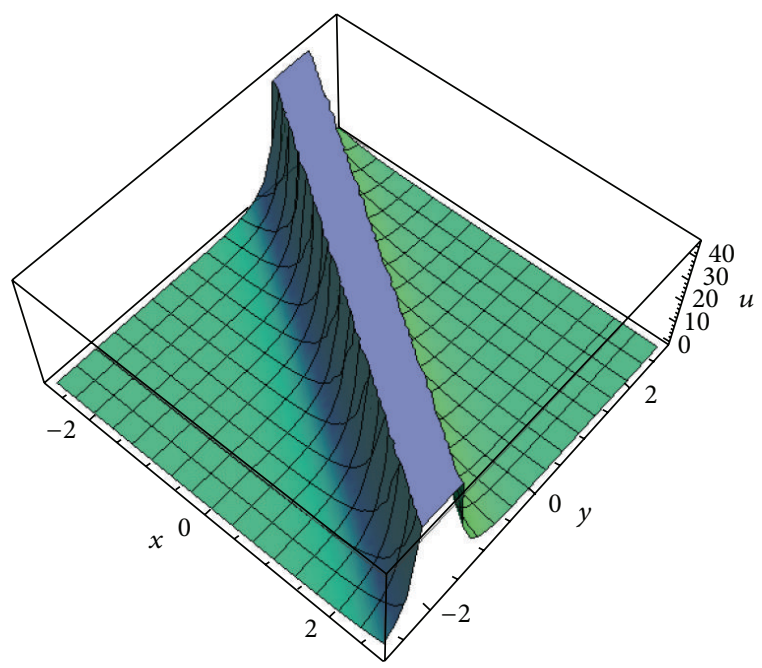

Figure 2: Profile of solution (26).

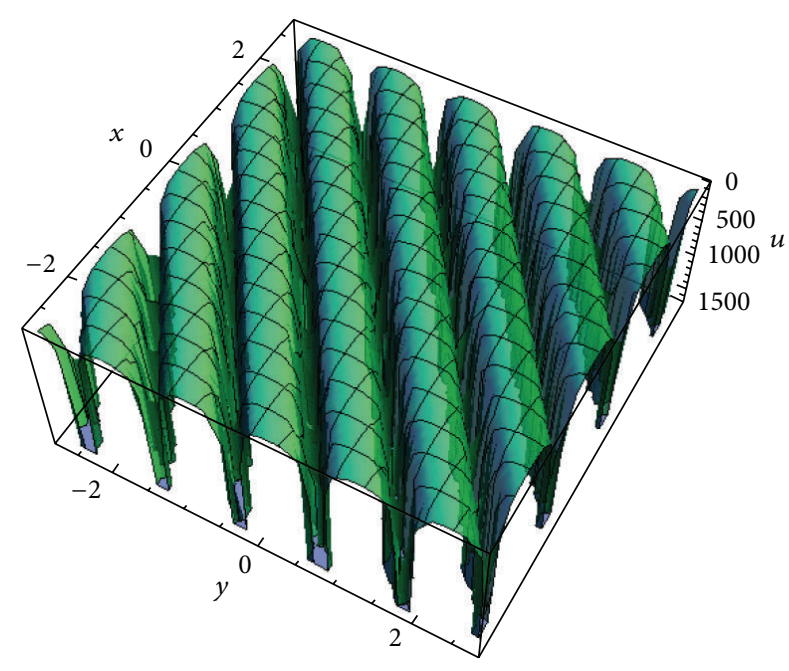

Figure 3: Profile of solution (27).

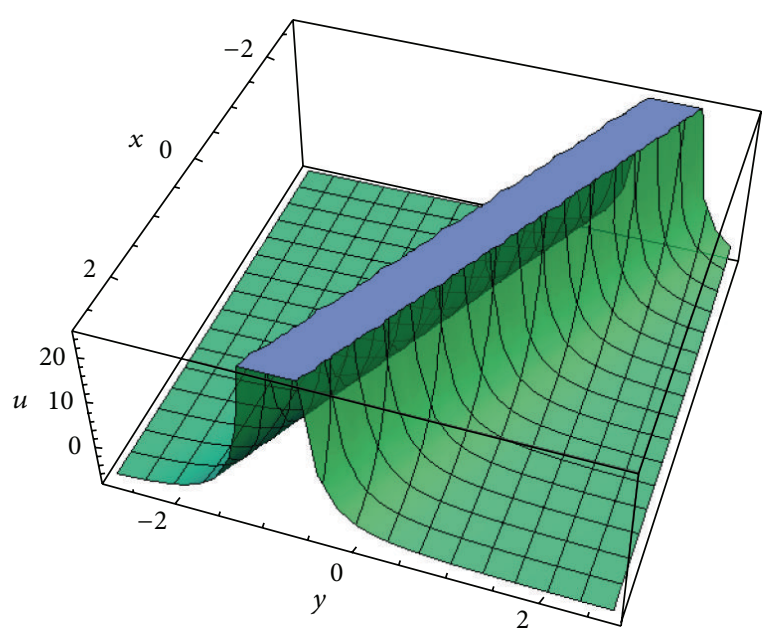

FIgURE 4: Profile of solution (28). 
Consider $n=3$. Again the application of the balancing procedure to fourth-order ODE yields $M=1$, so the solution of (11) is of the form

$$
F(z)=\mathscr{A}_{0}+\mathscr{A}_{1}\left(\frac{G^{\prime}(z)}{G(z)}\right) .
$$

Solving this system of algebraic equations with the aid of Maple, we obtain

$$
\begin{aligned}
\beta & =\frac{\alpha}{2} \mathscr{A}_{1}^{2}, \\
\mathscr{A}_{0} & =\frac{\lambda \sqrt{-a^{2} \gamma-2 a c \gamma+b^{2}-c^{2} \gamma}}{\sqrt{\alpha b^{2} \lambda^{2}-4 \alpha b^{2} \mu}}, \\
\mathscr{A}_{1} & =\frac{2 \sqrt{-a^{2} \gamma-2 a c \gamma+b^{2}-c^{2} \gamma}}{\sqrt{\alpha b^{2} \lambda^{2}-4 \alpha b^{2} \mu}} .
\end{aligned}
$$

Now using the general solution of (23) in (29), we have the following two types of travelling wave solutions of the KPMEW equation (1).

When $\lambda^{2}-4 \mu>0$, we obtain the hyperbolic function solution:

$$
\begin{aligned}
& u(x, y, t) \\
& \quad=\mathscr{A}_{0}+\mathscr{A}_{1}\left(-\frac{\lambda}{2}+\delta_{1} \frac{C_{1} \sinh \left(\delta_{1} z\right)+C_{2} \cosh \left(\delta_{1} z\right)}{C_{1} \cosh \left(\delta_{1} z\right)+C_{2} \sinh \left(\delta_{1} z\right)}\right),
\end{aligned}
$$

where $z=t-x-((a+c) / b) y, \delta_{1}=(1 / 2) \sqrt{\lambda^{2}-4 \mu}$, and $C_{1}$ and $C_{2}$ are arbitrary constants.

When $\lambda^{2}-4 \mu<0$, we obtain the trigonometric function solution:

$$
\begin{aligned}
& u(x, y, t) \\
& \quad=\mathscr{A}_{0}+\mathscr{A}_{1}\left(-\frac{\lambda}{2}+\delta_{2} \frac{-C_{1} \sin \left(\delta_{2} z\right)+C_{2} \cos \left(\delta_{2} z\right)}{C_{1} \cos \left(\delta_{2} z\right)+C_{2} \sin \left(\delta_{2} z\right)}\right),
\end{aligned}
$$

where $z=t-x-((a+c) / b) y, \delta_{2}=(1 / 2) \sqrt{4 \mu-\lambda^{2}}$, and $C_{1}$ and $C_{2}$ are arbitrary constants.

\section{Conservation Laws of (1)}

In this section we construct conservation laws for (1). The multiplier method $[29,30]$ will be used.

The zeroth-order multiplier $\Lambda(t, x, y, u)$ for (1) is given by

$$
\begin{aligned}
\Lambda= & -\frac{y^{3}}{6 \gamma} f_{1}^{\prime}(t)+x y f_{1}(t)-\frac{y^{2}}{2 \gamma} f_{2}^{\prime}(t) \\
& +x f_{2}(t)+y f_{3}(t)+f_{4}(t),
\end{aligned}
$$

where $f_{1}(t), f_{2}(t), f_{3}(t)$, and $f_{4}(t)$ are arbitrary functions of $t$. Corresponding to the above multiplier we have the following conserved vectors of (1):

$$
\begin{aligned}
& T_{1}^{t}=\frac{1}{24 \gamma}\left\{-12 \gamma y f_{1}(t) u-6 \beta \gamma y f_{1}(t) u_{x x}\right. \\
& +6 \beta \gamma x y f_{1}(t) u_{x x x}+12 \gamma x y f_{1}(t) u_{x} \\
& \left.-\beta y^{3} f_{1}^{\prime}(t) u_{x x x}-2 y^{3} f_{1}^{\prime}(t) u_{x}\right\} \text {, } \\
& T_{1}^{x}=-\frac{y}{24 \gamma u}\left\{4 \alpha n y^{2} f_{1}^{\prime}(t) u_{x} u^{n}-24 \alpha \gamma n x f_{1}(t) u_{x} u^{n}\right. \\
& -\beta y^{2} f_{1}^{\prime \prime}(t) u_{x x} u+3 \beta y^{2} f_{1}^{\prime}(t) u_{t x x} u \\
& +2 y^{2} f_{1}^{\prime}(t) u_{t} u-12 \beta \gamma f_{1}^{\prime}(t) u_{x} u \\
& +6 \beta \gamma x f_{1}^{\prime}(t) u_{x x} u+12 \beta \gamma f_{1}(t) u_{t x} u \\
& -18 \beta \gamma x f_{1}(t) u_{t x x} u-12 \gamma x f_{1}(t) u_{t} u \\
& +24 \alpha \gamma f_{1}(t) u^{n+1}-2 y^{2} f_{1}^{\prime \prime}(t) u^{2} \\
& \left.+12 \gamma x f_{1}^{\prime}(t) u^{2}\right\} \\
& T_{1}^{y}=\frac{1}{6}\left\{3 y^{2} f_{1}^{\prime}(t) u-6 \gamma x f_{1}(t) u\right. \\
& \left.+6 \gamma x y f_{1}(t) u_{y}-y^{3} f_{1}^{\prime}(t) u_{y}\right\} ; \\
& T_{2}^{t}=\frac{1}{8 \gamma}\left\{-4 \gamma f_{2}(t) u-2 \beta \gamma f_{2}(t) u_{x x}+2 \beta \gamma x f_{2}(t) u_{x x x}\right. \\
& \left.+4 \gamma x f_{2}(t) u_{x}-\beta y^{2} f_{2}^{\prime}(t) u_{x x x}-2 y^{2} f_{2}^{\prime}(t) u_{x}\right\} \text {, } \\
& T_{2}^{x}=-\frac{1}{8 \gamma u}\left\{4 \alpha n y^{2} f_{2}^{\prime}(t) u_{x} u^{n}-8 \alpha \gamma n x f_{2}(t) u_{x} u^{n}\right. \\
& -\beta y^{2} f_{2}^{\prime \prime} u_{x x} u+3 \beta y^{2} f_{2}^{\prime} u_{t x x} u \\
& +2 y^{2} f_{2}^{\prime}(t) u_{t} u-4 \beta \gamma f_{2}^{\prime}(t) u_{x} u \\
& +2 \beta \gamma x f_{2}^{\prime}(t) u_{x x} u+4 \beta \gamma f_{2}(t) u_{t x} u \\
& -6 \beta \gamma x f_{2}(t) u_{t x x} u-4 \gamma x f_{2}(t) u_{t} u \\
& +8 \alpha \gamma f_{2}(t) u^{n+1}-2 y^{2} f_{2}^{\prime \prime}(t) u^{2} \\
& \left.+4 \gamma x f_{2}^{\prime}(t) u^{2}\right\} \\
& T_{2}^{y}=\frac{1}{2}\left\{2 y f_{2}^{\prime}(t) u+2 \gamma x f_{2}(t) u_{y}-y^{2} f_{2}^{\prime}(t) u_{y}\right\} ; \\
& T_{3}^{t}=\frac{1}{4}\left\{\beta y f_{3}(t) u_{x x x}+2 y f_{3}(t) u_{x}\right\}, \\
& T_{3}^{x}=-\frac{y}{4 u}\left\{-4 \alpha n f_{3}(t) u_{x} u^{n}+\beta f_{3}^{\prime}(t) u_{x x} u-3 \beta f_{3}(t) u_{t x x} u\right. \\
& \left.-2 f_{3}(t) u_{t} u+2 f_{3}^{\prime}(t) u^{2}\right\} \\
& T_{3}^{y}=\gamma y f_{3}(t) u_{y}-\gamma f_{3}(t) u
\end{aligned}
$$




$$
\begin{gathered}
T_{4}^{t}=\frac{1}{4}\left\{\beta f_{4}(t) u_{x x x}+2 f_{4}(t) u_{x}\right\}, \\
T_{4}^{x}=-\frac{1}{4 u}\left\{-4 \alpha n f_{4}(t) u_{x} u^{n}+\beta f_{4}^{\prime}(t) u_{x x} u-3 \beta f_{4}(t) u_{t x x} u\right. \\
\left.-2 f_{4}(t) u_{t} u+2 f_{4}^{\prime}(t) u^{2}\right\}, \\
T_{4}^{y}=\gamma f_{4}(t) u_{y} .
\end{gathered}
$$

Remark. The presence of the arbitrary functions in the multiplier leads to a family of infinitely many conservation laws for (1).

\section{Concluding Remarks}

In this paper we obtained the solutions of a generalized two-dimensional nonlinear Kadomtsev-Petviashvilimodified equal width equation by employing the Lie group analysis, the optimal systems of one-dimensional subalgebras, and the $\left(G^{\prime} / G\right)$-expansion method. The solutions obtained are solitary waves and nontopological solitons. The conservation laws for the underlying equation were also derived by using the multiplier method.

\section{Conflict of Interests}

The authors declare that there is no conflict of interests regarding the publication of this paper.

\section{References}

[1] M. J. Ablowitz and P. A. Clarkson, Soliton, Nonlinear Evolution Equations and Inverse Scattering, Cambridge University Press, Cambridge, UK, 1991.

[2] M. Wang, Y. Zhou, and Z. Li, "Application of a homogeneous balance method to exact solutions of nonlinear equations in mathematical physics," Physics Letters A, vol. 216, no. 1-5, pp. 67-75, 1996.

[3] C. H. Gu, Soliton Theory and Its Application, Zhejiang Science and Technology Press, Zhejiang, China, 1990.

[4] Y. Chen and Z. Yan, "The Weierstrass elliptic function expansion method and its applications in nonlinear wave equations," Chaos, Solitons and Fractals, vol. 29, no. 4, pp. 948-964, 2006.

[5] V. B. Matveev and M. A. Salle, Darboux Transformation and Soliton, Springer, Berlin, Germany, 1991.

[6] J. Hu, "Explicit solutions to three nonlinear physical models," Physics Letters A, vol. 287, no. 1-2, pp. 81-89, 2001.

[7] J. Hu and H. Zhang, "A new method for finding exact traveling wave solutions to nonlinear partial differential equations," Physics Letters A, vol. 286, no. 2-3, pp. 175-179, 2001.

[8] R. Hirota, The Direct Method in Soliton Theory, vol. 155 of Cambridge Tracts in Mathematics, Cambridge University Press, Cambridge, UK, 2004.

[9] M. Wang, X. Li, and J. Zhang, “The $\left(G^{\prime} / G\right)$-expansion method and travelling wave solutions of nonlinear evolution equations in mathematical physics," Physics Letters A, vol. 372, no. 4, pp. 417-423, 2008.
[10] D. Lü, "Jacobi elliptic function solutions for two variant Boussinesq equations," Chaos, Solitons \& Fractals, vol. 24, no. 5, pp. 1373-1385, 2005.

[11] Z. Yan, "Abundant families of Jacobi elliptic function solutions of the $(2+1)$-dimensional integrable Davey-Stewartson-type equation via a new method," Chaos, Solitons and Fractals, vol. 18, no. 2, pp. 299-309, 2003.

[12] S.-Y. Lou and J. Lu, "Special solutions from the variable separation approach: the Davey-Stewartson equation," Journal of Physics, A: Mathematical and General, vol. 29, no. 14, pp. 4209-4215, 1996.

[13] A.-M. Wazwaz, "The tanh and the sine-cosine methods for compact and noncompact solutions of the nonlinear KleinGordon equation," Applied Mathematics and Computation, vol. 167, no. 2, pp. 1179-1195, 2005.

[14] Z. Yan, "The new tri-function method to multiple exact solutions of nonlinear wave equations," Physica Scripta, vol. 78, no. 3, Article ID 035001, 5 pages, 2008.

[15] Z. Yan, "Periodic, solitary and rational wave solutions of the 3D extended quantum Zakharov-Kuznetsov equation in dense quantum plasmas," Physics Letters, Section A: General, Atomic and Solid State Physics, vol. 373, no. 29, pp. 2432-2437, 2009.

[16] M. Wang and X. Li, "Extended F-expansion method and periodic wave solutions for the generalized Zakharov equations," Physics Letters A, vol. 343, no. 1-3, pp. 48-54, 2005.

[17] S. Zhang, "Application of Exp-function method to highdimensional nonlinear evolution equation," Chaos, Solitons and Fractals, vol. 38, no. 1, pp. 270-276, 2008.

[18] W.-X. Ma, T. Huang, and Y. Zhang, "A multiple exp-function method for nonlinear differential equations and its application," Physica Scripta, vol. 82, no. 6, Article ID 065003, 2010.

[19] G. W. Bluman and S. Kumei, Symmetries and Differential Equations, Applied Mathematical Sciences, Springer, New York, NY, USA, 1989.

[20] P. J. Olver, Applications of Lie Groups to Differential Equations, vol. 107 of Graduate Texts in Mathematics, Springer, New York, NY, USA, 2nd edition, 1993.

[21] L. V. Ovsiannikov, Group Analysis of Differential Equations, Academic Press, New York, NY, USA, 1982.

[22] N. H. Ibragimov, CRC Handbook of Lie Group Analysis of Differential Equations, vol. 1, CRC Press, Boca Raton, Fla, USA, 1994.

[23] N. H. Ibragimov, CRC Handbook of Lie Group Analysis of Differential Equations, vol. 2, CRC Press, Boca Raton, Fla, USA, 1995.

[24] N. H. Ibragimov, CRC Handbook of Lie Group Analysis of Differential Equations, vol. 3, CRC Press, Boca Raton, Fla, USA, 1996.

[25] K. R. Adem and C. M. Khalique, "Exact solutions and conservation laws of a $(2+1)$-dimensional nonlinear KP-BBM equation," Abstract and Applied Analysis, vol. 2013, Article ID 791863, 5 pages, 2013.

[26] A.-M. Wazwaz, "The tanh method and the sine-cosine method for solving the KP-MEW equation," International Journal of Computer Mathematics, vol. 82, no. 2, pp. 235-246, 2005.

[27] A. Saha, "Bifurcation of travelling wave solutions for the generalized KP-NEW equations," Communications in Nonlinear Science and Numerical Simulation, vol. 17, no. 9, pp. 3539-3551, 2012.

[28] M. Wei, S. Tang, H. Fu, and G. Chen, "Single peak solitary wave solutions for the generalized KP-MEW $(2,2)$ equation under 
boundary condition," Applied Mathematics and Computation, vol. 219, no. 17, pp. 8979-8990, 2013.

[29] S. C. Anco and G. Bluman, "Direct construction method for conservation laws of partial differential equations. Part I: examples of conservation law classifications," European Journal of Applied Mathematics, vol. 13, no. 5, pp. 545-566, 2002.

[30] K. R. Adem and C. M. Khalique, "Conservation laws and traveling wave solutions of a generalized nonlinear ZK-BBM equation," Abstract and Applied Analysis, vol. 2014, Article ID 139513, 5 pages, 2014. 


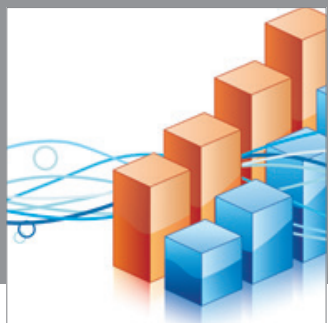

Advances in

Operations Research

mansans

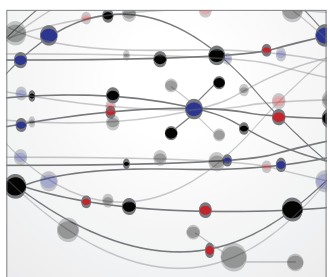

The Scientific World Journal
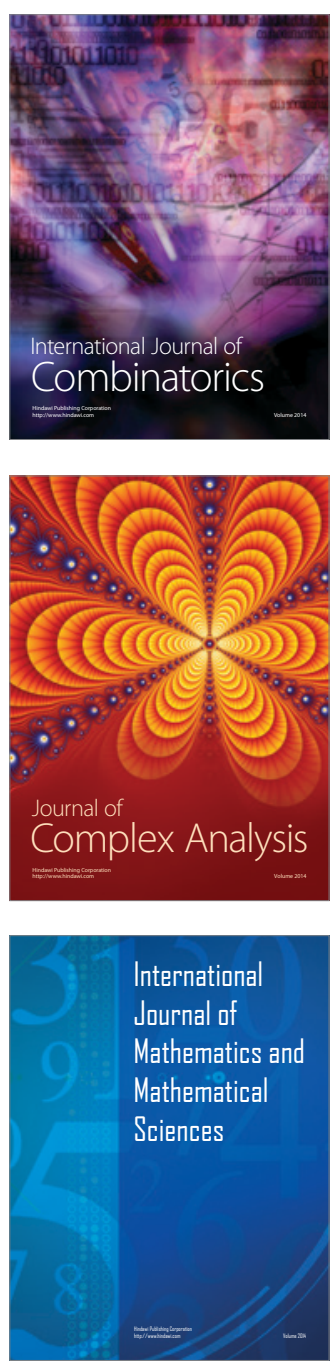
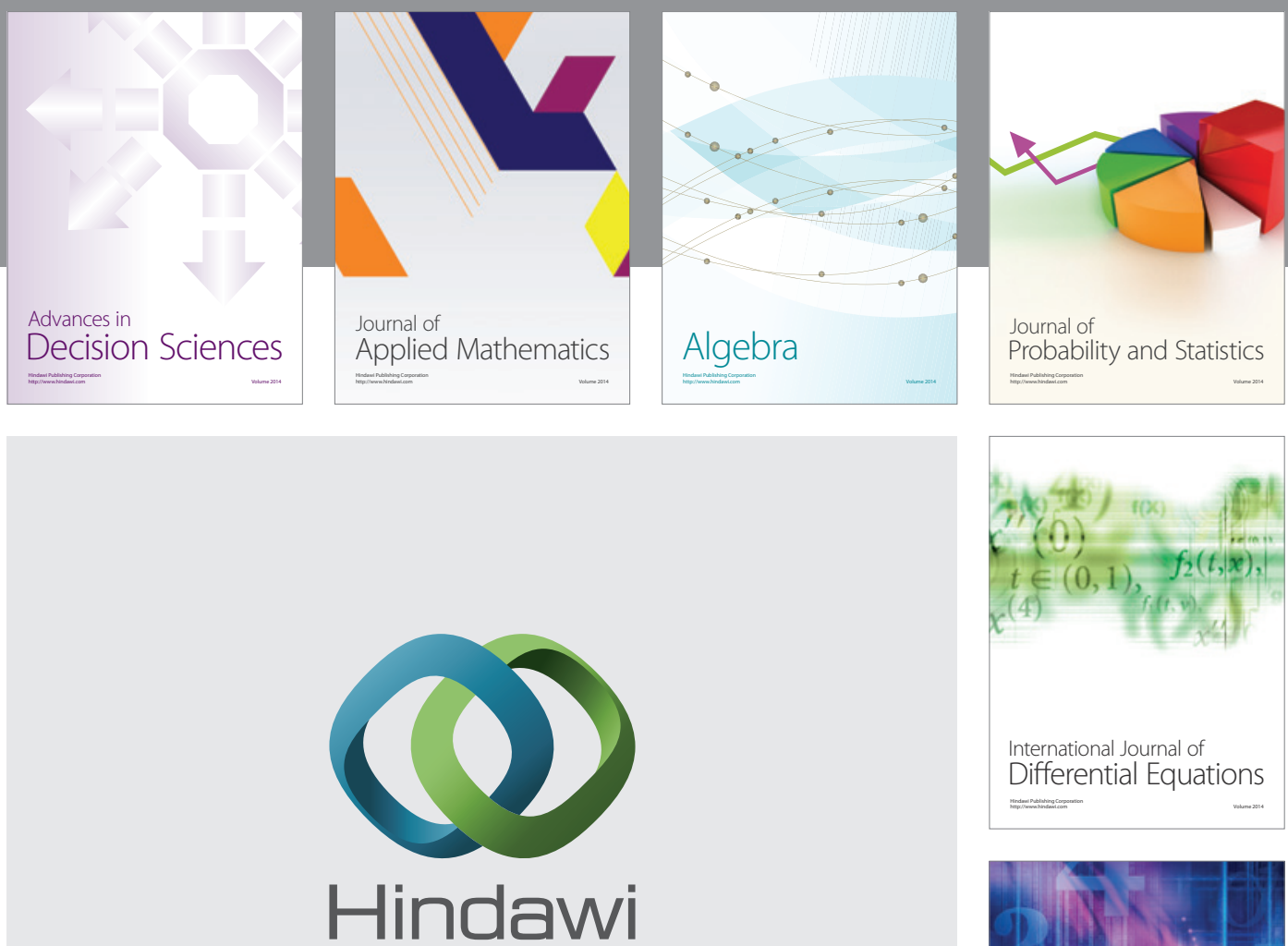

Submit your manuscripts at http://www.hindawi.com
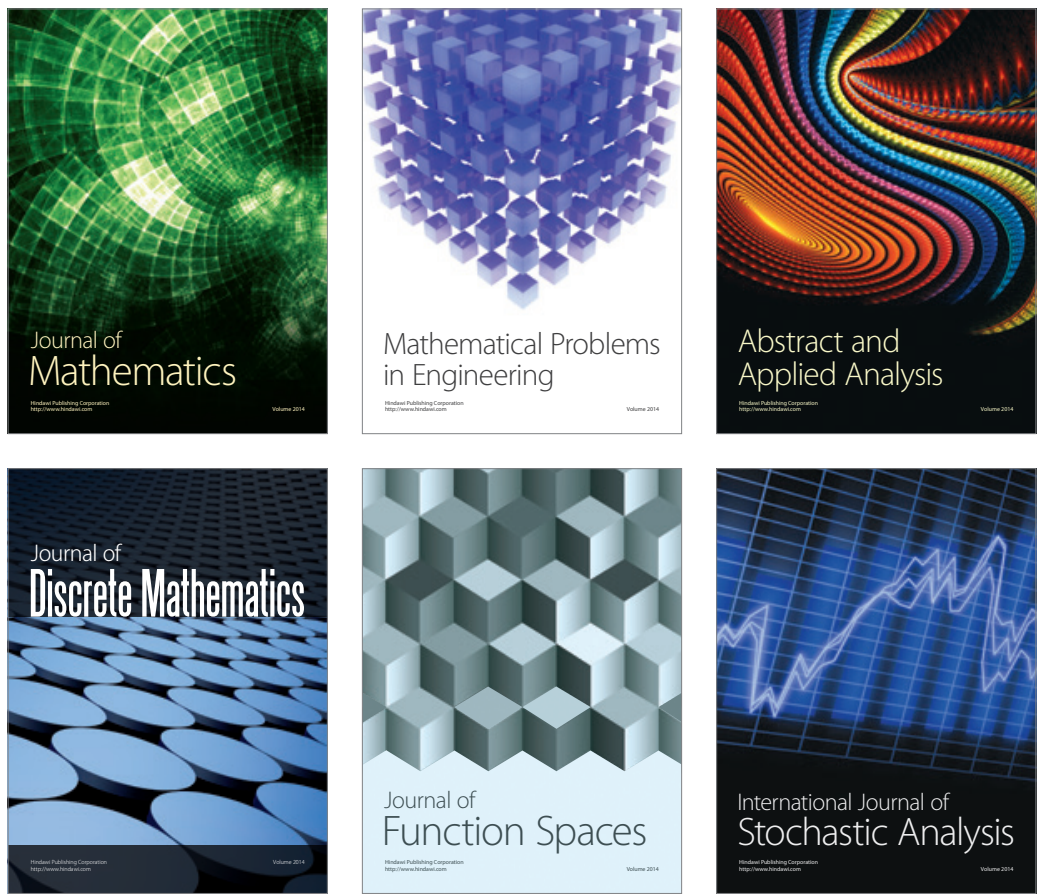

Journal of

Function Spaces

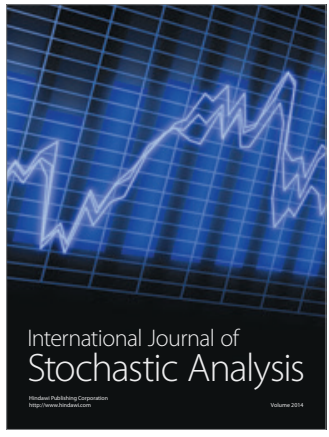

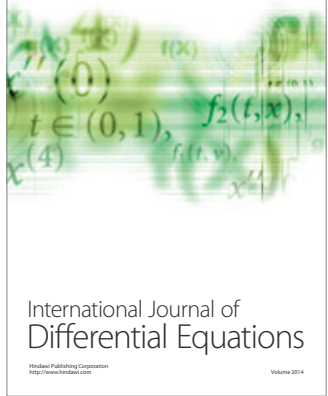
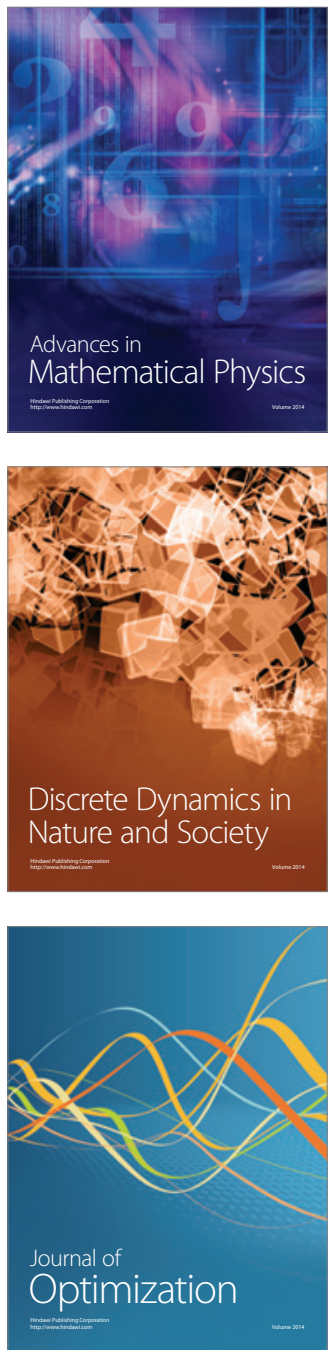